\title{
Cygnus OB2 at all wavelengths
}

\author{
Jürgen Knödlseder \\ Centre d'Etude Spatiale des Rayonnements, 9, avenue Colonel-Roche, \\ B.P. 4346, F-31028 Toulouse Cedex 4, la France
}

\begin{abstract}
The Cygnus X region is one of the most nearby star formation re gions within our Galaxy, that is recognised by prominent emission throughout the entire electromagnetic spectrum, from radio to gamma-ray waves. The centre is populated by the peculiar stellar cluster Cyg OB2, traditionally classified as $\mathrm{OB}$ association, but in the light of modern data more likely the prototype of a young globular cluster within our own Galaxy. Heavily shredded by dense molecular clouds this object has been less thoroughly studied than other, more distant star formation complexes in the Milky Way. I will review our current knowledge about this object, by exposing its properties and that of its environment throughout the entire electromagnetic spectrum. Particular emphasis will be given to infrared, radio-continuum, X-ray, and gamma-ray line observations that find in Cyg OB2 a text-book case for multiwavelength studies.
\end{abstract}

\section{The stellar population of Cygnus OB2}

\subsection{Optical surveys}

The history of optical observations of Cyg OB2 started in 1953, when Münch \& Morgan first noticed a clustering of eleven blue giants in the area northeast of $\gamma$ Cygni. Following this discovery, Johnson \& Morgan (1954) obtained spectroscopic observations of the eleven stars, and recognised the considerable interstellar absorption towards these objects, which made them to the most heavily obscured stars that were known at that epoch. From their data, they obtained a first distance estimate of $1.5 \mathrm{kpc}$ to the association, which is already close to the modern value of $1.7 \mathrm{kpc}$ (e.g., Massey \& Thompson 1991). Morgan et al. (1954a; 1954b) discovered seven further early type members, of which the first (Schulte 12) is the most famous object in Cyg OB2 because of its unequaled visual luminosity $\left(\mathrm{M}_{V} \simeq-10\right)$ and large extinction $\left(A_{V} \simeq 10\right)$. Schulte (1956a) named the association VICygni, and in the following years, he established a list of 31 association members from his initial list of 88 suspected OB stars (Schulte $1956 \mathrm{~b} ; 1958)$.

One of the most comprehensive studies of Cyg OB2 has been undertaken by Reddish et al. (1966) who performed detailed photographic $U B V$ investigations of the area. From star counts on their photographic plates down to a magnitude limit of $B=16.5$, they estimated a number of $\sim 400$ OB stars within the association. By extending the star counts to fainter magnitudes using the Palomar Sky Survey plates, they found a total of 3000 association members, for which however no spectral type information was available. Based on the luminosity 
Table 1. Parameters of Cygnus OB2

\begin{tabular}{ll}
\hline \hline centre (J2000) & $\alpha=20^{\mathrm{h}} 33^{\mathrm{m}} 10^{\mathrm{s}}, \delta=+41^{\circ} 12^{\prime}$ \\
distance & $1.7 \pm 0.2 \mathrm{kpc}$ \\
age & $3-4 \times 10^{6} \mathrm{yr}$ \\
diameter & $\sim 2^{\circ}(\sim 60 \mathrm{pc})$ \\
members earlier F3V & $8600 \pm 1300$ \\
OB star members & $2600 \pm 400$ \\
O-type star members & $120 \pm 20$ \\
total stellar mass & $4-10 \times 10^{4} \mathrm{M}_{\odot}$ \\
central mass density $\rho_{0}$ & $40-150 \mathrm{M}_{\odot} \mathrm{pc}^{-3}$ \\
IMF slope $\Gamma$ & $-1.0 \ldots-1.6$ \\
\hline
\end{tabular}

function, they estimated an association mass between $(5.8-27) \times 10^{3} \mathrm{M}_{\odot}$, where the lower limit corresponds to $400 \mathrm{OB}$ stars members while the upper limit assumes that all 3000 member stars were indeed of OB type. From the star counts they determined an elliptical shape for Cyg OB2 with major and minor axes of $48^{\prime} \times 28^{\prime}$, and they suggested that this shape might result from the rotation of the association around the minor axis which lies parallel to the galactic plane. On basis of the dimension, star numbers and total mass, Reddish et al. (1966) were also the first who suggested that Cyg OB2 may be regarded as a young globular cluster, similar to the blue globular clusters in the Magellanic Clouds.

No further extensive survey of Cyg OB2 has been performed until the early nineties, when Torres-Dodgen et al. (1991) obtained Strömgren and near-infrared photometry for about 80 stars in the association. Using the method of spectroscopic parallax, they estimated the distance of Cyg OB2 to $1.7 \pm 0.2 \mathrm{kpc}$, and by the means of isochrone fitting they estimated a minimum age of $3 \mathrm{Myr}$. The first CCD photometric survey of Cyg OB2 has been undertaken by Massey \& Thompson (1991) who also performed spectroscopic observations of 76 stars, thus enhancing by a factor of four the number of member stars with spectral type classification. They also determined the distance to $1.7 \mathrm{kpc}$, and they estimated the slope of the initial mass function to $\Gamma=-1.0 \pm 0.3$.

\subsection{Near-infrared surveys}

The first survey of Cyg OB2 in the near-infrared domain has been performed by Voelcker \& Elsässer (1973) who observed 80 stars in the $J-, H$-, $K$ - and $L$-bands. Based on their observations they suggest that the stars are still located in the natal dust cloud from which they have been formed. This idea is supported by near-infrared observations of Torres-Dodgen et al. (1991) from which the authors determined a normal extinction law with a ratio of total-to-selective extinction of $R_{V}=3.04 \pm 0.09$ (see also McMillan \& Tapia 1977 and references therein).

Using $J, H$, and $K_{S}$ photometric data from the 2MASS survey, Knödlseder (2000) re-determined the morphology and stellar content of Cyg OB2 and demonstrated that previous visual surveys were heavily biased by the foreground extinction pattern. In total, he found $120 \pm 20$ O-type star members and estimated the total cluster mass to $4-10 \times 10^{4} \mathrm{M}_{\odot}$. He determined the association diameter to $2^{\circ}$, which at a distance of $1.7 \mathrm{kpc}$ converts into a radius of $30 \mathrm{pc}$. Assuming spherical symmetry, this results in an average cluster density of $0.4-0.9 \mathrm{M}_{\odot} \mathrm{pc}^{-3}$, with a central density reaching $\rho_{\mathrm{c}}=40-150 \mathrm{M}_{\odot} \mathrm{pc}^{-3}$. The 2MASs data indicate 
a relatively steep slope of the initial mass function of $\Gamma=-1.6 \pm 0.1$. Taken all these parameters together, Knödlseder (2000) confirms the suggestion of Reddish et al. (1966) that Cyg OB2 should be classified as a young globular galactic cluster.

In a recent near-infrared spectroscopic survey of 46 candidate early-type members of Cyg OB2, Comerón et al. (2002) confirmed the findings of Knödlseder (2000). In particular, they detect $\mathrm{Br} \gamma$ emission lines from 20 stars which is characteristic for evolved stars that experience considerable mass loss. Most of the $\mathrm{Br} \gamma$ stars are redder than predicted by the reddening vector, indicating circumstellar dust emission that probably has been created by the mass ejections. This suggestion is substantiated by the detection of $\mathrm{H}_{2}$ and $\mathrm{CO}$ in some stars, which is indicative of abundant circumstellar molecular material. Comerón et al. (2002) estimate the fraction of $\mathrm{Br} \gamma$ emission line to O-type stars to $25 \%$, which places an interesting age limit on Cyg OB2 if we assume that all these stars are indeed in their Wolf-Rayet phase. From the evolutionary model presented in Figure 3 of Knödlseder et al. (2002), this fraction is reached at an age of 3-4 Myr under the assumption of coeval star formation, in agreement with findings by other methods (see Knödlseder et al. 2002 and references therein).

\subsection{Mid- and far-infrared observations}

Mid- and far-infrared observations of the Cygnus region have been conducted by balloon-borne telescopes (Campbell et al. 1980 and references therein), but the most comprehensive list of infrared sources in Cyg OB2 is based on observations with the IRAS satellite (e.g., Odenwald 1989). Most of the mid- and far-infrared point sources detected in the Cyg OB2 area are either embedded early-type stars or star clusters, or young stellar objects (YSO). Many of them are associated to thermal radio continuum sources which are suggestive of $\mathrm{H}$ II regions. Some of the point sources have been identified with known members, suggesting warm circumstellar dust which is attributed to extensive mass loss. Examples are stars \#5 and \#12, and the putative member MWC 349 (Parthasarathy et al. 1992).

Several tens of YSOs have been found in the area of Cyg OB2 (Parthasarathy et al. 1992; Odenwald \& Schwartz 1993) which may indicate that star formation is still ongoing in the association. Yet, although the density of YSOs is higher in the Cyg OB2 area with respect to adjacent sky fields, it is unclear whether the objects are indeed members of Cyg OB2 or whether they are situated in the surrounding matter, where the kinematical activity of Cyg OB2 has led to compression of the medium and possibly triggered star formation.

\subsection{Peculiar stars}

Cyg OB2 houses some of the hottest and most luminous stars known in our Galaxy. Stars \#7 and \#22-A are the only stars of the extreme type O3 If* known in the northern hemisphere (Walborn et al. 2002). Star \#5, also known as V729 Cyg, is an evolved contact binary system formed by a O7 Ia+Ofpe/WN9 pair that undergo considerable mass loss and mass exchange (Rauw et al. 1999). Star \#12 figures amongst the visually most luminous $\left(\mathrm{M}_{V} \simeq-10\right)$ and at the same time most obscured $\left(A_{V} \simeq 10\right)$ stars known within our Galaxy. It seems to violate the empirical Humphrey-Davidson upper-luminosity limit, which led Massey \& Thompson (1991) to the suggestion that it might be a Luminous Blue Variable (LBV) star in quiescence. Indeed, this star shows photometric and 
spectroscopic variability that is typical for LBV stars, and the large extinction may indicate the presence of circumstellar material that is a remain of some past episodic mass-loss outburst.

The presence of thermal and non-thermal components of radio emission in a couple of stars (\#8A, \#9 and WR 147) further indicates massive stars that undergo mass loss. Higgs et al. (1994) have identified a spherical shell in high resolution radio observations of G $79.29+0.46$, located at the south-western edge of Cyg OB2. They identified a central star of extreme luminosity $\left(M_{V} \simeq-10\right)$ and extinction $\left(A_{V} \simeq 16\right)$ at a distance compatible with that of Cyg OB2. More recent spectroscopy strongly supports their initial idea of G 79.29+0.46 being a LBV star, which possibly is in the transition of becoming a Wolf-Rayet star (Voors et al. 2000). The observed radio and infrared shell is probably due to $\sim 14 \mathrm{M}_{\odot}$ of circumstellar material that have been ejected by the star (Waters et al. 1996). Yet, the distance estimate is rather uncertain, and the derived radial velocity of $v_{\mathrm{LSR}}=-27 \mathrm{~km} \mathrm{~s}^{-1}$ would place the object rather in the Perseus spiral arm (Voors et al. 2000).

Three Wolf-Rayet stars in the Cyg OB2 field have so far been suggested as members of the association: WR 144, WR 145, and WR 146 (WR numbers and positions in the VIIth Galactic WR catalogue, van der Hucht 2001). WR 146 is a binary star (Niemela et al. 1998) that shows non-thermal radio emission located between both components that is strongly suggestive of wind-wind collision (Dougherty et al. 2000; Setia Gunawan et al. 2000). A similar situation has been observed in WR 147 (Setia Gunawan et al. 2001), yet it is situated slightly outside the $2^{\circ}$ diameter association boundary, and its suggested distance of $650 \mathrm{pc}$ (Morris et al. 2000) indicates a foreground object. Wind-wind collisions may also explain the high-energy gamma-ray emission from the unidentified GRO-EGRET source 3EG J2033+4118 that has tentatively been associated to star \#5 (Benaglia et al. 2001). Recently, even TeV gamma-ray emission has been observed near 3EG J2033+4118 and star \#5 (Rowell \& Horns 2002), indicating that particle acceleration to multi-TeV energies may take place at the shock front of the colliding wind regions.

The question of still on-going star formation in Cyg OB2 is closely related to the identification of pre-main-sequence members, and the peculiar star MWC 349A has indeed been suggested as such (Meyer et al. 2002 and references therein). MWC 349A possesses characteristics of a pre-main-sequence $\mathrm{B}[\mathrm{e}]$ star, in particular a dusty rotating circumstellar disk and a bipolar outflow, yet other authors argue that it could also be a massive $\mathrm{B}[\mathrm{e}]$ supergaint (e.g., White $\&$ Becker 1985). From the similarity of polarisation magnitude and position angle between MWC 349A and nearby Cyg OB2 members, Meyer et al. (2002) conclude that it is indeed part of the association.

Finally, the X-ray binary star Cyg X-3 lies in the field of Cyg OB2, yet distance estimates of about $\sim 9 \mathrm{kpc}$ (Predehl et al. 2000 ) place this object far behind the association, located either in the Perseus or the outer spiral arm. 


\section{The environment of Cygnus OB2}

\subsection{The distance problem}

Studying the environment of Cyg OB2 is intimately related to the assignment of distances to adjacent structures and stellar groups which then allows to dress a three-dimensional picture of the region and to study the interplay of the various constituents. However, kinematic distance estimates in the Cygnus region are unreliable for distances below $4 \mathrm{kpc}$ because the velocity gradient due to differential galactic rotation is substantially smaller than the typical velocity of streaming or expansion motions of a few $10 \mathrm{kms}^{-1}$. The only reasonably well defined distances come from spectroscopic parallax measurements towards stars, yet also here the patchy extinction pattern and the possible presence of circumstellar dust may considerably deteriorate the estimates. Although there is little doubt about the existence of a spiral arm feature in this region of the sky that is seen tangentially, part of the apparent alignment of objects along the line of sight at distances from 0.5 to $4 \mathrm{kpc}$ may result from erroneous distance estimates. Thus, in order to understand the Cyg OB2 environment, estimates of distance uncertainties are crucial, and should be considered seriously.

\subsection{Cygnus $X$}

Cyg OB2 lies near the centre of an extended $\left(\sim 10^{\circ}\right)$ radio emission structure known as Cygnus X (Piddington \& Minnett 1952). Cygnus X is composed of numerous individual H II regions, which have first been listed by Downes \& Rinehart (1968). Dickel et al. (1969) estimated their distance by comparing their radio-continuum intensity to the $\mathrm{H} \alpha$ surface brightness of corresponding optical nebula, and found that most of them are situated in a narrow distance range of $1.2-2.4 \mathrm{kpc}$, with a typical distance uncertainty of $25 \%$. Cong (1977) identified two molecular cloud complexes that are associated with the H II regions: the DR 21 complex east of Cyg OB2 which comprises the radio sources DR 18, DR 20, DR 21, DR 22, and DR 23, and the IC 1318bc complex west of Cyg OB2 which comprises the radio sources DR6, DR 9, DR 12, DR 13, DR 15, and IC $1318 \mathrm{bc}$. These complexes are perceptible at various wavelengths (radio continuum, infrared, molecular gas) as the most prominent emission regions of Cygnus X with Cyg OB2 matched in between. Distance estimates to these complexes are $1.6 \pm 0.4 \mathrm{kpc}$ for DR 21 (Comerón \& Torra 1999) and $1.5 \pm 0.4 \mathrm{kpc}$ for IC 1318 bc (Dickel et al. 1969), which within the uncertainties is compatible with the distance to Cyg OB2.

Véron (1965) has suggested that Cygnus $\mathrm{X}$ is a single giant $\mathrm{H}$ II region that is powered by Cyg OB2, yet the identification of individual $\mathrm{H}$ II regions with their exciting stars in Cygnus X indicates that there are also other ionising sources in the area. Wendker (1970) has identified a diffuse thermal radio component that constitutes $\gtrsim 50 \%$ of the emission in Cygnus, which may indeed present the extended Strömgren sphere of Cyg OB2. Using an evolutionary synthesis model to predict the multi-wavelength emission of the massive star populations in the Cygnus X region, Knödlseder et al. (2002) confirm this hypothesis. About $50 \%$ of the predicted ionising luminosity in the area comes from Cyg OB2 while the rest is provided by massive stars that are found in the surrounding associations

and clusters. Using their predicted Lyman continuum flux of $10^{51} \mathrm{ph} \mathrm{s}^{-1}$ for Cyg OB2, and assuming an electron temperature and density of $T_{\mathrm{e}}=6000 \mathrm{~K}$ and 
$n_{\mathrm{e}}=10 \mathrm{~cm}^{-3}$, respectively, a Strömgren sphere with a radius of $60 \mathrm{pc}$ is expected, corresponding to an angular diameter of $4^{\circ}$ at a distance of $1.7 \mathrm{kpc}$. This is comparable to the size of the diffuse radio emission component, which shows two maxima at a distance of $\sim 2^{\circ}$ from Cyg OB2 that coincide with the DR 21 and IC 1318bc complexes (Wendker 1970). Interestingly, it has been suggested that the HII region observed near the mass-losing giant NML Cyg, situated at about $2.7^{\circ}$ from the centre of $\mathrm{Cyg} \mathrm{OB} 2$, results from the photo-ionisation of the stellar outflow by UV photons from Cyg OB2 (Morris \& Jura 1983). Thus, the Strömgren sphere of Cyg OB2 may even reach a radius of $\sim 80 \mathrm{pc}$.

\subsection{Wind swept shells}

Wind swept shells around Cyg OB2 have been searched and tentatively identified by several authors (e.g., Kaftan-Kassim 1961; Heiles 1979; Gosachinskii et al. 1999; Lozinskaya et al. 2002), yet no convincing detection has been made so far. The major difficulty in finding interstellar shells in the Cygnus region arises from the fact that for distances of up to $4 \mathrm{kpc}$, the differential galactic rotation results only in low radial velocities $|v| \lesssim 10 \mathrm{~km} \mathrm{~s}^{-1}$, a value that is smaller than the dispersion of interstellar gas velocities $\left(\sim 15 \mathrm{~km} \mathrm{~s}^{-1}\right)$ in the Galaxy (e.g., Heiles 1979; Geballe et al. 1999). Consequently, all the matter of the local spiral arm piles-up in a narrow radial velocity interval which completely blends the possible signature of an expanding shell.

Lozinskaya et al. (2002) estimate the mechanical luminosity of Cyg OB2 to a few $10^{39} \mathrm{erg} \mathrm{s}^{-1}$ during the past 2 million years. Assuming a typical initial density of $100 \mathrm{~cm}^{-3}$, in agreement with measurements of immersed molecular clumps in Cyg OB2 by Gredel \& Münch (1994), the size of a bubble created by the winds of $\mathrm{Cyg}$ OB2 should amount to $63 \mathrm{pc}$, with a bubble shell velocity of $19 \mathrm{~km} \mathrm{~s}^{-1}$ (Weaver et al. 1977). At a distance of $1.7 \mathrm{kpc}$, this should result in a shell diameter of $4^{\circ}$, comparable to the estimated size of the Strömgren sphere. Indeed, the DR 21 and IC $1318 \mathrm{bc}$ complexes lie at the approximate location of the hypothesised wind blown shell, and the numerous compact radio sources, YSOs, and embedded young clusters that are found in these complexes (Le Duigou \& Knödlseder 2002) suggest that Cyg OB2 may have triggered star formation in their interiors.

\subsection{The Cygnus $X$-ray superbubble}

The Cygnus X-ray superbubble has been discovered by Cash et al. (1980) as an uncomplete ring of soft X-ray emission $13^{\circ} \times 18^{\circ}$ in diameter surrounding the Cygnus $\mathrm{X}$ region. The morphology of the $\mathrm{X}$-ray emission is shaped by heavy foreground extinction due to the Great Cygnus Rift, and the underlying source could in reality present a nearly uniform emission morphology (Cash et al. 1980; Uyaniker et al. 2001).

The origin of the Cygnus X-ray superbubble is still a puzzle, and the protagonists split into two groups who either suggest a single superbubble formed by $\mathrm{Cyg}$ OB2 or a superposition of numerous sources that are superimposed along the line of sight of the local spiral arm. Cash et al. (1980), belonging to the first group, suggested that a series of 30-100 supernovae exploding over a period of $3-10 \mathrm{Myr}$ in a former stellar generation of the Cyg OB2 association may have created the structure. Yet, there is no evidence for the existence of such a former generation of stars in Cyg OB2, and Abbott et al. (1981) proposed that the 
Table 2. Massive young galactic star clusters.

\begin{tabular}{lccccc}
\hline \hline cluster & $\begin{array}{c}d \\
(\mathrm{kpc})\end{array}$ & $\begin{array}{c}t_{\text {age }} \\
(\mathrm{Myr})\end{array}$ & $N$ (O-type stars) & $\begin{array}{c}\log M \\
\left(\mathrm{M}_{\odot}\right)\end{array}$ & $\begin{array}{c}\text { diameter } \\
(\mathrm{pc})\end{array}$ \\
\hline Central & 8 & $\sim 5$ & $\sim 100$ & 4.0 & 2 \\
Arches & 8 & $2.5 \pm 0.5$ & $\sim 160$ & 4.3 & 0.4 \\
Quintuplet & 8 & $4 \pm 1$ & $\sim 100$ & 3.8 & 2 \\
W 43 & $\sim 7$ & & $\sim 100$ & & 5 \\
W 49A & $11.4 \pm 1.2$ & $\$ 2$ & $\sim 100$ & & 15 \\
Cyg OB2 & $1.7 \pm 0.2$ & $3-4$ & $120 \pm 20$ & $4.6-5.0$ & 60 \\
NGC 3603 & $7 \pm 1$ & $\sim 3$ & $\sim 50$ & 3.7 & 10 \\
Westerlund 1 & $1.1 \pm 0.4$ & $4-5$ & & $4.5-5.0$ & 5 \\
\hline \hline
\end{tabular}

combined stellar winds from Cyg OB2 members may have formed the bubble during the last 2 million years. On the other hand, Bochkarev \& Sitnik (1985) summarise arguments against the single superbubble scenario and argue that the $\mathrm{X}$-ray emission results from discrete sources in the various $\mathrm{OB}$ associations and clusters that are found in the Cygnus region. In particular, the X-ray superbubble seems to be elongated along the galactic plane which is contrary to the expectation for an expanding bubble which should expand more easily perpendicular to the galactic disk. Hence, similar to the reasoning for the Cygnus $\mathrm{X}$ radio emission, the superbubble is probably a result of the superposition of various objects along the line of sight, yet a major contribution from the stellar wind activity of Cyg OB2 members is expected.

\subsection{Gamma-ray line emission}

Prominent $1.809 \mathrm{MeV}$ gamma-ray line emission has been reported from the Cygnus X region based on observations of the GRO-COMPTEL telescope (del Rio et al. 1996; Plüschke et al. 2002). The $1.809 \mathrm{MeV}$ gamma-ray line arises from the decay of ${ }^{26} \mathrm{Al}$, a radioactive isotope with a mean lifetime of about one million years. ${ }^{26} \mathrm{Al}$ is mainly produced during the core hydrogen burning phase in massive stars, and is subsequently ejected by stellar winds (in particular during the LBV and Wolf-Rayet phases) and/or supernova explosions. The presence of ${ }^{26} \mathrm{Al}$ in the Cygnus region is again a clear indicator of extensive mass loss by massive $\left(M>20 \mathrm{M}_{\odot}\right)$ stars in this area. Using an evolutionary synthesis model, Knödlseder et al. (2002) have suggested that Cyg OB2 is indeed the dominant ${ }^{26} \mathrm{Al}$ source in the Cygnus $\mathrm{X}$ region. Yet, actual models fail to explain the observed $1.809 \mathrm{MeV}$ line intensity, indicating possible shortcomings of nucleosynthesis calculations in massive stars that might be related to neglecting stellar rotation and/or binarity (Knödlseder et al. 2002).

\section{Galactic context}

Cyg OB2 is not the only massive young star cluster that has been observed in the Milky Way. The list of such objects amounts to at least eight clusters in the Galaxy (Table 2), and encloses the Central, Arches, and Quintuplet clusters (Figer, these Proceedings), W 43 (Blum et al. 1999), W 49A (Conti \& Blum 
2002), NGC 3603 (Moffat et al. 2002; Moffat et al. these Proceedings), and Westerlund 1 (Negueruela \& Clark, these Proceedings). These clusters are characterised by typical Lyman continuum luminosities of about $10^{51} \mathrm{ph} \mathrm{s}^{-1}$ which corresponds to an equivalent of about $\sim 100$ O-type stars. Consequently, they are all very young, with ages of a few Myr, and some, such as W 49A, are still embedded in their natal cloud. This leads to the question why no older massive star clusters have been observed so far, with ages of say a few 10-100 Myr. Probably it is observationally difficult to unveil them since the absence of ionising stars won't lead to a prominent thermal radio emission feature, optical H II region, or $1.809 \mathrm{MeV}$ gamma-ray line signature, that triggered the discovery of some of the known clusters. On the other hand, at least in the galactic centre, cluster disruption time scales are of the order of $\lesssim 10 \mathrm{Myr}$ (Kim et al. 1999), and at least the three known clusters in this area should disrupt within the next million years.

Some of the clusters, such as the Arches Cluster, show a considerable mass segregation (Stolte et al. 2002), others do not, e.g., Cyg OB2 (Knödlseder 2000). Also, the radial extent of the clusters vary by about two orders of magnitudes, and given that they have roughly similar mass (at least within one order of magnitude) their density varies by over six orders of magnitudes! This large spectrum certainly tells us something about the cluster formation process, which may have been different for the different clusters. Future multi-wavelength observations should shed more light on these clusters and their environments, and will hopefully unveil the conditions that lead to the formation of these localised starbursts within our Galaxy.

\section{References}

Abbott, D.C., Bieging, J.H., Churchwell, E. 1981, ApJ 250, 645

Benaglia, P., Romero, G.E., Stevens, I.R., Torres, D.F. 2001, A\&A 366, 605

Blum, R.D., Damineli, A., Conti, P.S. 1999, ApJ 117, 1392

Bochkarev, N.G., Sitnik, T.G. 1985, Ap\&SS 108, 237

Campbell, M.F., Hoffmann, W.F., Thronson, H.A.Jr., Harvey, P.M. 1980, ApJ 238, 122

Cash, W., Charles, P., Bowyer, S., et al. 1980, ApJ (Letters) 238, L71

Comerón, F., Torra, J. 1999, A\&A 349, 605

Comerón, F., Pasquali, A., Rodighiero, G., Stanishev, V., De Filippis, E., López Martí, B., Gálvez Ortiz, M.C., Stankov, A., Gredel, R. 2002, A\&A 389, 874

Cong, H.-L. 1977, A Survey of CO in Cygnus X, PhD thesis, Columbia University, USA Conti, P.S., Blum, R.D. 2002, ApJ 564, 827

del Río, E., von Ballmoos, P., Bennett, K., Bloemen, H., Diehl, R., Hermsen, W., Knödlseder, J., Oberlack, U., Ryan, J., Schönfelder, V., Winkler, C. 1996, A\&A 315, 237

Dickel, H.R., Wendker, H., Bieritz, J.H. 1969, A\&A 1, 270

Downes, D., Rinehart, R. 1968, ApJ 144, 937

Dougherty, S.M., Williams, P.M., Pollacco, D.L. 2000, MNRAS 316, 143

Geballe, T.R., McCall, B.J., Hinkle, K.H., Oka, T. 1999, ApJ 510, 251

Gosachinskii, I.V., Lozinskaya, T.A., Pravdikova, V.V. 1999, Astron. Reports 43, 391

Gredel, R., Münch, G. 1994, A\&A 285, 640

Heiles, C. 1979, ApJ 229, 533 
Higgs, L.A., Wendker, H.J., Landecker, T.L. 1994, A\&A 291, 295

van der Hucht, K.A. 2001, New Astron. Reviews 45, 135

Johnson, H.L., Morgan, W.W. 1954, ApJ 119, 344

Kaftan-Kassim, M.A. 1961, ApJ 133, 821

Kim, S.S., Morris, M., Lee, H.M. 1999, ApJ 525, 228

Knödlseder, J. 2000, A\&A 360, 539

Knödlseder, J., Cerviño, M., Le Duigou, J.-M., Meynet, G., Schaerer, D., von Ballmoos, P. 2002, A\&A 390, 945

Le Duigou, J.-M., Knödlseder, J. 2002, A\&A 392, 869

Lozinskaya, T.A., Pravdikova, V.V., Finoguenov, A.V. 2002, Astron. Letters 28, 223

Massey, P., Thompson, A.B. 1991, AJ 101, 1408

McMillan, R.S., Tapia, S. 1977, ApJ 212, 714

Meyer, J.M., Nordsieck, K.H., Hoffman, J.L. 2002, ApJ 123, 1639

Moffat, A.F.J., Corcoran, M.F., Stevens, I.R., et al. 2002, ApJ 573, 191

Morgan, W.W., Johnson, H.L., Roman, N.G. 1954a, PASP 66, 85

Morgan, W.W., Meinel, A.B., Johnson, H.L. 1954b, ApJ 120, 506

Morris, P.M., van der Hucht, K.A., Crowther, P.A., Hillier, D.J., Dessart, L., Williams, P.M., Willis, A.J. 2000, A\&A 353, 624

Morris, M., Jura, M. 1983, ApJ 267, 179

Münch, L., Morgan, W.W. 1953, ApJ 118, 161

Niemela, V.S., Shara, M.M., Wallace, D.J., Zurek, D.R., Moffat, A.F.J. 1998, ApJ 115, 2047

Odenwald, S.F., Schwartz, P.R. 1993, ApJ 405, 706

Odenwald, S.F. 1989, AJ 97, 801

Parthasarathy, M., Jain, S.K., Bhatt, H.C. 1992, A\&A 266, 202

Piddington, J.H., Minnett, H.C. 1952, Aust. J. Sci. Res. 17, 1952

Plüschke, S., Cerviño, M., Diehl, R., Kretschmer, K., Hartmann, D.H., Knödlseder, J. 2002, New Astron. Reviews 46, 535

Predehl, P., Burwitz, V., Paerels, F., Trümper, J. 2000, A\&A (Letters) 357, L25

Rauw, G., Vreux, J.-M., Bohannan, B. 1999, ApJ 517, 416

Reddish, V.C., Lawrence, L.C., Pratt, N.M. 1966, Publ. R. Obs. Edinburgh 5, 111

Rowell, G.P., Horns, D. 2002, in: The Gamma-Ray Universe, Proc. XXII Moriond Astrophysics Meeting, Les Arc, Savoie, France, March 2002, in press

Schulte, D.H. 1956a, ApJ 123, 250

Schulte, D.H. 1956b, ApJ 124, 530

Schulte, D.H. 1958, ApJ 128, 41

Setia Gunawan, D.Y.A., de Bruyn, A.G., van der Hucht, K.A., Williams, P.M. 2000, A\&A 356, 676

Setia Gunawan, D.Y.A., de Bruyn, A.G., van der Hucht, K.A., Williams, P.M. 2001, A\&A 368, 484

Stolte, A., Grebel, E.K., Brandner, W., Figer, D.F. 2002, A\&A 394, 459

Torres-Dodgen, A.V., Tapia, M., Carroll, M. 1991, MNRAS 249, 1

Uyaniker, B., Fürst, E., Reich, W., Aschenbach, B., Wielebinski, R. 2001, A\&A 371, 675

Véron, P. 1965, Annales d'Astrophysique 28, 391 
Voelcker, K., Elsässer, H. 1973, in: J. M. Greenberg, H.C. van der Hulst (eds.), Interstellar Dust and Related Topics, Proc. IAU Symposium No. 52 (Dordrecht: Kluwer), p. 529

Voors, R.H.M., Geballe, T.R., Waters, L.B.F.M., Najarro, F., Lamers, H. 2000, A\&A 362, 236

Walborn, N.R., Howarth, I.D., Lennon, D.J., Massey, P., Oey, M.S., Moffat, A.F.J., Skalkowski, G., Morrell, N.I., Drissen, L., Parker, J.Wm. 2002, ApJ 123, 2754

Waters, L.B.F.M., Izumiura, H., Zaal, P.A., Geballe, T.R., Kester, D.J.M., Bontekoe, T.R. 1996, A\&A 313, 866

Weaver, R., McCray, R., Castor, J., Shapiro, P., Moore, R. 1977, ApJ 218, 377

Wendker, H.J. 1970, A\&A 4, 378

White, R.L., Becker, R.H. 1985, ApJ 297, 677

\section{Discussion}

ConTI: The size of Cyg OB2 seems quite a bit larger than its energetic countcrparts, e.g., R 136, NGC 3603, the GC clusters, W 49A. Any comment?

KNÖDLSEDER: This is indeed interesting and may (should) be related to the way how Cyg OB2 has been formed.

KUDRITZKI: You seem to be concerned that by doubling the number of O-type stars you produce too many Lyman continuum photons. But the nebula could be optically thin, at least partially, in the Lyman continuum and there must be dust absorption.

KNÖDLSEDER: Indeed, but our actual estimates are already situated at the upper limit of the observations, hence doubling the number of O-type stars would push it probably too much (in particular, our analysis of near-IR data of the area does not indicate such a missing population of massive stars.)

STEVENS: Do the $\gamma$-ray results provide any constraints on stellar evolution or stellar evolution models?

KNÖDLSEDER: Indeed, this was our starting point! By comparing the $\gamma$-ray data to the stellar populations in Cygnus, we make inferences about nucleosynthesis in massive stars (see Knödlseder et al. 2002). It turns out that actual nucleosynthesis models under-produce ${ }^{26} \mathrm{Al}$, and we believe that this is related to additional convection and posssibly the effects of stellar rotation.

EL EID: One should be careful with ${ }^{26} \mathrm{Al}$ production in massive stars, because of the many sources involved. In addition, advanced phases of massive stars may contribute to its production. So, one should be careful not to put the main emphasis on rotation.

KNÖDLSEDER: It seems that the main sequence evolution is what we see contributing and this seems to require enhancement of the production of ${ }^{26} \mathrm{Al}$, eventually thus to the effect of rotation on the main sequence.

CoRCoran: Do you expect any contribution by Cyg X-3 to the $\gamma$-ray emission?

KNÖDlSEDER: As far as I know, Cyg X-3 is situated far beyond Cyg OB2 in the Perseus arm, hence it should not be related to the cluster. No $\gamma$-ray-line emission is expected from this source, but it is a hard X-ray and soft $\gamma$-ray continuum emitter. 